\title{
Individual- and Regional-level determinants of Human Papillomavirus (HPV) vaccine refusal: the Ontario Grade 8 HPV vaccine cohort study
}

\author{
Olivia Remes ${ }^{1}$, Leah M Smith², Beatriz E Alvarado-Llano ${ }^{1}$, Lindsey Colley ${ }^{1}$ and Linda E Lévesque ${ }^{1 *}$
}

\begin{abstract}
Background: Studies on the determinants of human papillomavirus (HPV) vaccine use have generally focused on individual-level characteristics, despite the potentially important influence of regional-level characteristics. Therefore, we undertook a population-based, retrospective cohort study to identify individual- and regional-level determinants of HPV vaccine refusal (non-receipt) in Ontario's (Canada) Grade 8 HPV Immunization Program.

Methods: Ontario's administrative health and immunization databases were used to identify girls eligible for free HPV vaccination in 2007-2011 and to ascertain individual-level characteristics of cohort members (socio-demographics, vaccination history, health care utilization, medical history). The social and material characteristics of the girl's region (health unit) were derived from the 2006 Canadian Census. Generalized estimating equations (binomial distribution, logit link) were used to estimate the population-average effects of individual- and regional-level characteristics on HPV vaccine refusal.
\end{abstract}

Results: Our cohort consisted of 144,047 girls, $49.3 \%$ of whom refused HPV vaccination. Factors associated with refusal included a previous diagnosis of Down's syndrome $(\mathrm{OR}=1.37,95 \% \mathrm{Cl} 1.16-1.63)$ or autism $(\mathrm{OR}=1.60,95 \% \mathrm{Cl} 1.34-1.90)$, few physician visits $(\mathrm{OR}=1.45,95 \% \mathrm{Cl} 1.35-1.55)$, and previous refusal of mandatory $(\mathrm{OR}=2.23,95 \% \mathrm{Cl} 2.07-2.40)$ and optional $(\mathrm{OR}=3.96,95 \% \mathrm{Cl}$ 3.87-4.05) vaccines. Refusal was highest among the lowest and highest income levels. Finally, a previous diagnosis of obesity and living in an area of high deprivation were associated with lower refusal ( $O R=0.87,95 \% \mathrm{Cl} 0.83-0.92$ and $\mathrm{OR}=0.82$ 95\%, Cl 0.79-0.86, respectively).

Conclusions: Studies on HPV vaccine determinants should consider regional-level factors. Efforts to increase HPV vaccine acceptance should include vulnerable populations (such as girls of low income) and girls with limited contact with the healthcare system.

Keywords: Human papillomavirus, HPV vaccine, Cohort studies, Vaccine coverage, Vaccine hesitancy, Cohort studies

\section{Background}

The human papillomavirus (HPV) is the most common sexually transmitted infection worldwide, with an estimated $75 \%$ of all women having at least one HPV infection during their lifetime [1]. One of the most common manifestation of HPV infections is genital warts, with a prevalence of approximately $1-4 \%$ among young, sexually active women [2]. Importantly, the presence of anogenital warts can lead to significant physical, emotional, and social

\footnotetext{
* Correspondence: linda.levesque@queensu.ca

'Department of Public Health Sciences, Queen's University, 21 Arch Street, Room 313, Kingston, Ontario, Canada

Full list of author information is available at the end of the article
}

problems for those affected [3]. While genital warts inflict the greatest HPV-related burden on youth, HPV infections may also lead to cancers of the anogenital tract in adulthood, the most frequent being cervical cancer. Of the over 100 types of HPV that have been identified, HPV types 6 and 11 cause $90 \%$ of cases of genital warts [4] and types 16 and 18 cause $70 \%$ of cases of cervical cancer worldwide $[5,6]$. Overall, infections with these four HPV types represent a significant burden of illness and distress, and are estimated to cost the Canadian healthcare system \$33 million annually [7].

In 2006, the quadrivalent HPV (qHPV) vaccine was approved for use in Canada, the United States, Europe, and 
elsewhere [8]. In view of the vaccine's high efficacy, and the public health implications of HPV-related diseases, the Canadian government allocated $\$ 300$ million to the provinces and territories to fund the first three years of a national, school-based HPV vaccination program [9]. Despite widespread anticipation about the vaccine's potential to reduce the burden of anogenital warts and cervical cancer, $\mathrm{HPV}$ vaccine coverage was generally lower than anticipated in Canada, reaching nationwide lows of 50\% in Alberta and Manitoba, 53\% in Ontario, and 65\% in British Columbia $[10,11]$. High refusal of the HPV vaccine has also been documented in a number of European and Indo-Pacific countries, the United States, and some Central and South American countries [12].

Vaccine refusal falls within the spectrum of a growing phenomenon recently referred to as "vaccine hesitancy" [13]. Decision-making with respect to health behaviours, including vaccination, has been shown to be complex, and influenced by both individual- and regional-level factors $[14,15]$. Indeed, the World Health Organization's (WHO) Strategic Advisory Group of Experts (SAGE) on Immunization recently highlighted the importance of contextual factors including socio-cultural and environmental ones, as determinants of vaccine hesitancy [13]. Yet, studies of the determinants of HPV vaccine uptake published to date have focused predominantly on individual-level characteristics, with little consideration for the potentially important influence that an individual's environment can have on the decision-making process [16,17]. Given that low vaccine coverage undermines public health efforts aimed at reducing the burden of HPV-related diseases, and may threaten the cost-effectiveness of HPV vaccination programs, we undertook a population-based, retrospective cohort study of girls eligible for Ontario's Grade 8 HPV vaccination program to identify the individual- and health unit-level determinants of HPV vaccine refusal.

\section{Methods}

This study was approved by the Research Ethics Board of Queen's University and Sunnybrook Health Sciences Centre.

\section{Ontario's Grade $8 \mathrm{HPV}$ vaccination program}

Ontario's HPV vaccination program was initiated in September of 2007. This voluntary program offers three doses of the vaccine free-of-charge to all Grade 8 girls in the province. These doses are generally administered by Public Health Nurses at school clinics in September/ October, November/December, and March/April to correspond with the recommended 0-, 2-, and 6-month dosing schedule of the vaccine. Although the vast majority of doses are given at these school clinics, eligible girls may also receive the vaccine through their public health unit or family physician. At the time of this study, eligible girls had until the end of their Grade 9 year to complete the vaccination series, provided they initiate it in Grade 8 [17]. All doses of the HPV vaccine are documented in the Immunization Record Information System (IRIS) database, regardless of where the dose is administered. Over 80,000 girls are eligible for this program each year.

\section{Data sources and record linkage}

Four administrative health databases were used for this study: (1) the Registered Persons Database (RPDB) to identify the birth cohorts and obtain information on socio-demographics, (2) the Canadian Institute for Health Information's Discharge Abstract Database (CIHI-DAD) for dates of hospital admissions and discharge diagnoses, (3) the National Ambulatory Care Reporting System (NACRS) for information on emergency department visit dates and diagnoses, and (4) the Ontario Health Insurance Plan (OHIP) database for information on fee-for-service claims submitted by physicians, including service dates and diagnoses. In these databases, each Ontario resident covered by the province's universal health insurance plan is represented by a unique encrypted identifier that enables complete record linkage at the level of the individual across databases and over time. These databases are continuously updated and can be accessed through the Institute for Clinical Evaluative Sciences' (ICES) satellite unit located at Queen's University. Described elsewhere in detail [18-22], these databases have been used extensively in health research, including studies of the HPV vaccine [23,24].

To obtain vaccination history, we used the IRIS database, which was developed by the Ministry of Health and LongTerm Care (MOHLTC) to assist the province's 36 health units in tracking and recording immunizations of schoolaged children, as required under the Immunization of School Pupils Act (1982) [25]. This database is also used to document the receipt of optional vaccines, including the $\mathrm{HPV}$ vaccine. Indeed, the IRIS database has been shown to accurately capture information on HPV vaccination status with a sensitivity of $99.8 \%$ (95\% CI: 99.3 - 99.9) and specificity of $97.7 \%$ (95\% CI: 96.3 - 98.7) [26]. At the time of this study, the IRIS database of 21 health units had been transferred to ICES and were available for our use.

We also used data from the 2006 Canadian Census, a mandatory, self-reported survey conducted every five years by Statistics Canada to enumerate all residents of Canada and provide a socio-demographic portrait of the Canadian population. We used the Census database to ascertain socio-demographic information at the level of the health unit (i.e., regional-level characteristics) [27].

\section{Study design and population}

We identified a retrospective cohort of girls eligible for Ontario's Grade $8 \mathrm{HPV}$ vaccination program between 
2007/08 and 2010/11 using the province's administrative health records. Because a girl's school grade was not available in these data holdings, birth cohorts were used to identify the eligible population. Since students typically turn thirteen by December 31 of their Grade 8 school year, girls born in 1994, 1995, 1996 and 1997, would have been in Grade 8 in September 2007, 2008, 2009, and 2010, respectively, and would have been eligible for the corresponding year's vaccination program. Although this approach could miss girls who were advanced or held back a grade, a re-abstraction study of a medium-sized health unit demonstrated that this birth cohort definition correctly identified $96.4 \%$ of eligible girls [28]. Girls whose vaccination records were not available at the time of the analysis (i.e., IRIS data not yet transferred to ICES for record linkage) were excluded from the cohort. Cohort members were followed from September 1 of their Grade 8 year until their date of death or March 31, 2011 (study end).

\section{HPV vaccination status}

The outcome of interest was HPV vaccine non-receipt (which we refer to as "refusal"), which was obtained from the IRIS database. A girl was classified as a "refuser" if she received no doses of the HPV vaccine; otherwise she was classified as an "acceptor".

\section{Individual-level characteristics}

We determined the socio-demographics, vaccination history, medical history, and health care utilization history of cohort members using the administrative health and immunization databases previously described. The RPDB was used to obtain information on age, sex, neighbourhood income quintile, and urban/rural residency at cohort entry.

Vaccination history was derived from the IRIS database and included vaccinations received from birth to cohort entry. The optional vaccines under consideration were hepatitis $\mathrm{B}$ and meningococcal conjugate because they are both offered in Grade 7 through a publicly funded, schoolbased program. Mandatory vaccines included the measles, mumps, rubella (MMR) vaccine, as well as the diphtheria, polio, and tetanus (DTP) vaccine. Vaccination history, particularly with optional vaccines, was used as a proxy for parental/guardian beliefs and attitudes toward vaccines in general.

Medical and health care utilization histories of cohort members were obtained between birth and cohort entry using the physician services (OHIP), emergency department (NACRS), and hospital (CIHI-DAD) databases. The medical conditions considered included those resulting in frequent contact with the health care system, as well as those serious enough to potentially affect the decision to vaccinate against HPV (e.g., autoimmune disorders, cancer, congenital anomalies, heart disease, neurological disease;
Additional file 1). Health care utilization was determined based on the number of outpatient physician visits, emergency department visits, hospital admissions, and in-patient length of stay prior to cohort entry. The frequency of each type of utilization was categorized based on the frequency distribution of the data. These categories were used as indicators of an individual's health status and their propensity to use the health care system.

\section{Characteristics of the health units}

The 2006 Canadian Census was used to obtain information on the social and material characteristics of the health unit within which a cohort member resided at cohort entry. We used information from the 2006 Census since this represented the latest data available prior to cohort entry (i.e., preceding the decision to vaccinate). Since health units are responsible for the administration and delivery of the HPV vaccination program in Ontario, the health unit was chosen as the regional level of interest for this study. Based on a review of the literature on the determinants of HPV vaccine acceptance, we initially considered 19 census variables (Additional file 2). We examined the variability of these characteristics across health units, the collinearity between variables, and each variable's univariable association with the outcome. Based on these analyses, we determined that only six of these characteristic warranted further consideration - average income, level of education, employment/population ratio, marital status, and living alone.

To further address the potential for collinearity between indicators, as well as to reduce the number of variables contributing to the model (given the limited number of health units contributing to the analysis), we developed an area deprivation index. The index was modelled after the Pampalon social and material deprivation index [29] and was derived using principal component analysis (PCA) [30]. Although we included the same six social and material factors contained in the Pampalon index in our PCA, only five loaded as a component/dimension and were thus included in our index; single parenthood was not considered further (Additional file 3). A score was calculated for each health unit based on the factor loadings of each of the five indicators. For the purposes of analysis, these scores were subsequently categorized into quartiles.

\section{Statistical analysis}

To estimate the population average effect of the determinants of interest on HPV vaccine refusal, we used generalized estimating equations (GEE) to account for the correlation introduced by the clustering of individuals within health units. The characteristics of the health unit were attributed to the girl, making her the unit of analysis. Since the outcome was dichotomous (refusal $v s$. non-refusal), and the correlation between subjects in 
health units was assumed to be equal, we used GEE with a logit link and an exchangeable correlation structure to estimate odds ratios (ORs) and 95\% confidence intervals (CIs; based on robust standard errors). The final model was selected using backward selection and a significance threshold of 0.1 for variable retention.

\section{Results}

Within the 21 health units, we identified 144,047 girls eligible for Ontario's Grade 8 HPV vaccination program between 2007/08 and 2010/11. Girls were a mean of 13.2 years of age at cohort entry and were followed for an average of 2.6 years. Approximately 3\% of cohort members refused MMR and/or DTP vaccination and $42 \%$ refused hepatitis B and/or meningococcal conjugate vaccination (Table 1). Almost $65 \%$ of girls resided in areas of low material and social deprivation.

The average number of cohort members per health unit was 6859 (range: 1012-18171). Overall, 49.3\% $(n=71,048)$ of cohort members refused HPV vaccination during the study period; refusal varied from $41.8 \%$ to $60.3 \%$ across health units. While absolute differences were small, neighbourhood income quintile was a statistically significant determinant of HPV vaccine refusal, with the highest level of refusal in the highest and lowest quintiles (Table 2). Immunization history was also a determinant of HPV vaccination in that HPV vaccine refusal was more common among girls who had not received optional and mandatory immunizations in the past $(\mathrm{OR}=3.96,95 \%$ CI 3.87-4.05; $\mathrm{OR}=2.23,95 \%$ CI $2.07-2.40$, respectively). Refusal was generally similar across categories of health care utilization and history of a serious medical condition, but there were some exceptions. In particular, there was a dose-response trend with respect to physician visits, whereby girls with the lowest level of contact with a physician were the most likely to refuse HPV vaccination $(\mathrm{OR}=1.45,95 \% \mathrm{CI} 1.35-1.55)$ and refusal decreased with increasing levels of physician visits $(\mathrm{p}<0.05)$. In addition, parents/guardians of girls with a history of autism or Down's syndrome appeared more likely to refuse HPV vaccination than caregivers with unaffected children ( $\mathrm{OR}=1.60,95 \%$ CI 1.34-1.90; OR $=1.37,95 \% \mathrm{CI}$ 1.16-1.63, respectively). In contrast, obesity was associated with decreased refusal (OR $=0.87,95 \%$ CI 0.83-0.92). Finally, health unit-level social and material deprivation was associated with HPV vaccine decision-making, with the lowest refusal observed among girls living in regions with the highest level of deprivation (OR $=0.82,95 \%$ CI $0.79,0.86$ ).

\section{Discussion}

Nearly half of all girls eligible for Ontario's free HPV vaccination program between the 2007/08 and 2010/11 school year did not receive the vaccine. Our study showed that factors related to this high level of refusal included previous diagnoses of Down's syndrome or autism, as well as low income and high income, and infrequent visits to the doctor. Interestingly, obesity and high area deprivation were determinants of HPV vaccine acceptance. Not surprisingly, a history of vaccine refusal was also associated with refusal of the HPV vaccine.

This study is one of the first to consider medical history as a potential determinant of HPV vaccine use. We found that a history of an obesity-related diagnosis was associated with increased HPV vaccine acceptance. As studies indicate there is an important association between obesity and cervical cancer [31], these results are promising as they suggest that this high risk group is receiving protection against cervical cancer. Nevertheless, given then limited information on this topic, these results need to be confirmed.

This study also found that girls with Down's syndrome or autism were less likely to receive the HPV vaccine than girls who have not been diagnosed with these intellectual disabilities (IDs). However, we used birth year to estimate the girls' grade 8 school year, and since individuals with IDs are more likely to start school late or be held back school grades [32], it is possible the association we observed merely reflects the fact that these girls were not yet eligible for publicly funded vaccination. Some individuals with IDs may be homeschooled [33], which would limit their access to the school-based vaccination program. Moreover, there is a common misconception that individuals with IDs are less sexually active than their peers [34,35], which may lead caregivers to perceive HPV immunization as less necessary for these children. In reality, however, adolescents with IDs report similar ages of sexual onset and rates of sexual activity as their typically developing peers $[34,35]$ and are at higher risk of sexual abuse [36], suggesting they are at a similar (if not higher) risk of HPV-related illnesses. As a result, this association should be further investigated. Regardless, our study highlights the importance of considering medical history in studies on the HPV vaccine as potential determinants of HPV vaccine use and as potential confounders of the effects of HPV vaccination.

We also found that HPV vaccine acceptance increased with increased levels of outpatient contacts with a physician. These results are consistent with studies from the United States that demonstrate the important, positive influence physicians can have on increasing HPV vaccine acceptance among parents and women $[16,37,38]$. Our study suggests that this relationship holds even in the context of a publicly funded, school-based program, where the vaccine is primarily delivered outside of the physician's office. Nevertheless, it is important to recognize that increased number of physician visits may also be a proxy for parental health beliefs and behaviours, which likely also influence $\mathrm{HPV}$ vaccine receipt.

Our study is also one of the first to consider both individual-level and regional-level characteristics as 
Table 1 Baseline characteristics of cohort members ( $N=144,047$ )

Characteristic
Individual-level characteristics
Socio-demographics
Income quintiles
$1^{\text {st }}$ (Low)
$2^{\text {nd }}$
$3^{\text {rd }}$
$4^{\text {th }}$
$5^{\text {th }}$ (High)
Missing
Place of residence
Rural
Urban
Missing
Vaccination history

Refusal of mandatory vaccines ${ }^{*}$

Refusal of optional vaccines ${ }^{\dagger}$

Health care utilization

Hospitalizations

$\leq 1$

2-4

$\geq 4$

Inpatient hospital stay (days)

$\leq 2$

3-1)

$\geq 11$

Emergency department visits

None

$1-4$

$5-12$

$\geq 13$

Outpatient physician visits

$\leq 42$

43-116

117-206

$\geq 207$

\section{Medical history}

Congenital anomalies

Viral diseases

Cardiovascular disease

Obesity

Autism

Mental illness

Neurological diseases
Frequency (\%)

21,357 (15.0)

25,689 (17.8)

30,823 (21.4)

32,495 (22.6)

30,803 (21.4)

$2,880(2.0)$

21,301 (14.8)

119,866 (83.2)

$2,880(2.0)$

4,160 (2.9)

$60,447(42.0)$

110,167 (76.5)

29,350 (20.4)

$4,530(0.03)$

103,292 (71.7)

38,359 (26.6)

2,396 (1.7)

48,413 (33.6)

$69,796(48.5)$

$21,229(14.7)$

4,609 (3.2)

$36,861(25.6)$

74,797 (51.9)

$26,794(18.6)$

$5,595(3.9)$

4,723 (3.3\%)

95,070 (66.0\%)

$26,620(18.5 \%)$

$6,264(4.4 \%)$

599 (0.42\%)

$44,588(31.0 \%)$

$12,972(9.0 \%)$
Table 1 Baseline characteristics of cohort members ( $N=144,047)$ (Continued)

\begin{tabular}{ll}
\hline Down's syndrome & $639(0.4 \%)$ \\
Diseases of the musculoskeletal system & $48,670(33.8 \%)$ \\
Respiratory disease & $63,250(43.9 \%)$ \\
Diseases of the endocrine system & $5,460(3.8 \%)$ \\
In-situ carcinoma and benign lesions & $13,649(9.5 \%)$ \\
Cancer & $2,491(1.7 \%)$ \\
Malnutrition & $6,558(4.5 \%)$ \\
Immune-mediated disorders & $100,874(70.0 \%)$ \\
Health unit-level characteristics & \\
Index of area deprivation (quartiles) & \\
$\quad 1^{\text {st }}$ (low) & $93,093(64.6)$ \\
$2^{\text {nd }}$ & $21,249(14.6)$ \\
$3^{\text {rd }}$ & $18,482(12.8)$ \\
$\quad 4^{\text {th }}$ (high) & $11,223(7.8)$ \\
\hline${ }^{*}$ Includes measles, mumps, rubella (MMR) and diphtheria, tetanus, pertussis \\
$\begin{array}{l}\text { (DTP) vaccines. } \\
{ }^{+} \text {Includes hepatitis B and meningococcal conjugate vaccines. }\end{array}$
\end{tabular}

potential independent predictors of HPV vaccination. Interestingly, we found that girls living in areas of high deprivation were more likely to receive the HPV vaccine than girls living in areas of low deprivation. Our results are not consistent with studies from the United States, which report that indicators of deprivation (e.g., low income, low education) are major barriers to HPV vaccination [16]. The latter findings are not surprising given the high cost of the vaccine series ( $\$ 450 \mathrm{CND})$ and the lack of publicly funded vaccination, but are of particular public health importance as these indicators of deprivation are also major risk factors for cervical cancer [39,40]. Accordingly, our results suggest the publicly funded, school-based nature of the HPV vaccination programs in Canada is helping to reduce inequities in access to the HPV vaccine that may ultimately help reduce inequities in HPV-related illnesses. Further studies are needed to corroborate these findings.

Importantly, our findings on area deprivation were independent of our one individual-level component of deprivation - family income. Indeed, income was associated with HPV vaccine refusal, whereby girls in the highest and lowest income quintiles were the most likely to refuse HPV vaccination. Our finding of high refusal among higher income families is consistent with a study from British Columbia (Canada) that found that parents of higher socio-economic status (SES) were less likely to accept having their daughters vaccinated, despite being more informed about the benefits of the HPV vaccine [41]. Similarly, studies have consistently reported that low income is associated with high refusal [16]. However, most of these studies were conducted in 
Table 2 Determinants of HPV vaccine refusal

\begin{tabular}{|c|c|c|c|}
\hline Characteristic & Refusal $n$ (\%) & Unadjusted OR (95\% Cl) & Adjusted $^{\dagger}$ OR $(95 \% \mathrm{Cl})$ \\
\hline \multicolumn{4}{|c|}{ Individual-level characteristics } \\
\hline \multicolumn{4}{|l|}{ Income quintiles ${ }^{*}$} \\
\hline $1^{\text {st }}$ (low) & $21,357(51.6)$ & $1.24(1.20,1.29)$ & $1.13(1.09,1.17)$ \\
\hline $2^{\text {nd }}$ & $25,689(47.3)$ & $1.04(1.01,1.08)$ & $1.01(0.98,1.05)$ \\
\hline $3^{\text {rd }}$ (reference) & $30,823(46.2)$ & 1.00 & 1.00 \\
\hline $4^{\text {th }}$ & $32,495(48.3)$ & $1.09(1.05,1.12)$ & $1.12(1.08,1.15)$ \\
\hline $5^{\text {th }}$ (high) & $30,803(49.7)$ & $1.15(1.11,1.18)$ & $1.21(1.17,1.25)$ \\
\hline \multicolumn{4}{|l|}{ Vaccination history } \\
\hline \multicolumn{4}{|c|}{ Refusal of mandatory vaccines } \\
\hline No (reference) & $139,887(48.5)$ & 1.00 & 1.00 \\
\hline Yes & $4,160(78.8)$ & $3.95(3.66,4.26)$ & $2.23(2.07,2.40)$ \\
\hline \multicolumn{4}{|c|}{ Refusal of optional vaccines } \\
\hline No (reference) & $83,600(34.8)$ & 1.00 & 1.00 \\
\hline Yes & $60,447(69.4)$ & $4.25(4.16,4.35)$ & $3.96(3.87,4.05)$ \\
\hline \multicolumn{4}{|c|}{ Health care utilization } \\
\hline \multicolumn{4}{|l|}{ Hospitalizations } \\
\hline$\leq 1$ (reference) & $110,167(48.3)$ & 1.00 & 1.00 \\
\hline $2-4$ & $29,350(49.5)$ & $0.95(0.93,0.98)$ & $0.94(0.91,0.97)$ \\
\hline$\geq 4$ & $4,530(52.6)$ & $1.13(1.07,1.20)$ & $0.96(0.90,1.03)$ \\
\hline \multicolumn{4}{|c|}{ Emergency department visits } \\
\hline None (reference) & $48,413(50.7)$ & 1.00 & 1.00 \\
\hline $1-4$ & $69,796(48.9)$ & $0.93(0.91,0.95)$ & $1.03(1.01,1.06)$ \\
\hline $5-12$ & $21,229(47.9)$ & $0.89(0.87,0.92)$ & $1.02(0.98,1.06)$ \\
\hline$\geq 13$ & $4,609(47.3)$ & $0.87(0.82,0.93)$ & $0.99(0.93,1.06)$ \\
\hline \multicolumn{4}{|c|}{ Outpatient physician visits } \\
\hline$\leq 42$ & $36,861(55.3)$ & $1.55(1.46,1.64)$ & $1.45(1.35,1.55)$ \\
\hline $43-116$ & $74,797(47.9)$ & $1.15(1.09,1.22)$ & $1.24(1.17,1.32)$ \\
\hline $117-206$ & $26,794(46.1)$ & $1.07(1.01,1.14)$ & $1.12(1.05,1.19)$ \\
\hline$\geq 207$ (reference) & $5,595(44.4)$ & 1.00 & 1.00 \\
\hline \multicolumn{4}{|l|}{ Medical history } \\
\hline \multicolumn{4}{|l|}{ Congenital anomalies } \\
\hline No (reference) & $139,324(49.3)$ & 1.00 & 1.00 \\
\hline Yes & $4,723(50.2)$ & $1.04(0.98,1.10)$ & $1.03(0.97,1.10)$ \\
\hline \multicolumn{4}{|l|}{ Viral diseases } \\
\hline No (reference) & $48,977(52.4)$ & 1.00 & 1.00 \\
\hline Yes & $95,070(47.8)$ & $0.83(0.81,0.85)$ & $0.96(0.94,0.99)$ \\
\hline \multicolumn{4}{|l|}{ Cardiovascular disease } \\
\hline No (reference) & $117,427(49.5)$ & 1.00 & 1.00 \\
\hline Yes & $26,620(48.5)$ & $0.96(0.93,0.98)$ & $1.03(1.00,1.06)$ \\
\hline \multicolumn{4}{|l|}{ Obesity } \\
\hline No (reference) & $137,783(49.5)$ & 1.00 & 1.00 \\
\hline Yes & $6,264(45.0)$ & $0.83(0.79,0.88)$ & $0.87(0.83,0.92)$ \\
\hline
\end{tabular}


Table 2 Determinants of HPV vaccine refusal (Continued)

\begin{tabular}{|c|c|c|c|}
\hline \multicolumn{4}{|l|}{ Autism } \\
\hline No (reference) & $143,448(49.3)$ & 1.00 & 1.00 \\
\hline Yes & $599(64.3)$ & $1.85(1.57,2.19)$ & $1.60(1.34,1.90)$ \\
\hline \multicolumn{4}{|l|}{ Mental illness } \\
\hline No (reference) & $99,459(49.2)$ & 1.00 & 1.00 \\
\hline Yes & $44,588(49.5)$ & $1.01(0.99,1.04)$ & $1.04(1.01,1.07)$ \\
\hline \multicolumn{4}{|l|}{ Neurological disorder } \\
\hline No (reference) & $131,075(49.3)$ & 1.00 & 1.00 \\
\hline Yes & $12,972(49.7)$ & $1.01(0.98,1.05)$ & $1.06(1.02,1.10)$ \\
\hline \multicolumn{4}{|l|}{ Down's syndrome } \\
\hline No (reference) & $143,408(49.3)$ & 1.00 & 1.00 \\
\hline Yes & $639(60.6)$ & $1.58(1.35,1.85)$ & $1.37(1.16,1.63)$ \\
\hline \multicolumn{4}{|c|}{ Health unit-level characteristics } \\
\hline \multicolumn{4}{|c|}{ Index of area deprivation (quartiles) } \\
\hline $1^{\text {st }}$ (low) (reference) & $93,093(49.8)$ & 1.00 & 1.00 \\
\hline $2^{\text {nd }}$ & $21,249(48.9)$ & $0.97(0.94,0.99)$ & $1.02(0.99,1.06)$ \\
\hline $3^{\text {rd }}$ & $18,482(49.2)$ & $0.98(0.95,1.01)$ & $1.01(0.97,1.05)$ \\
\hline $4^{\text {th }}$ (high) & $11,223(46.4)$ & $0.87(0.84,0.91)$ & $0.82(0.79,0.86)$ \\
\hline
\end{tabular}

OR: odds ratio; $95 \% \mathrm{Cl}$ : $95 \%$ confidence interval.

"Of the 2,880 girls with a missing postal code (and, consequently, income quintile), $92 \%$ were classified as refusers. This high percentage most likely reflects emigration from Ontario rather than actual refusal.

${ }^{\dagger}$ Adjusted for all other factors listed in the table.

the United States where important financial and access barriers exist. Since financial and access barriers are greatly reduced in countries, like Canada, that offer the HPV vaccine free of charge, our findings suggest that low income may be a proxy for other factors that influence vaccine acceptance in this population. Nevertheless, given the increased vulnerability of girls from low income families to HPV-related diseases (as described above), our findings demonstrate that this continues to be an important group for targeted cervical cancer risk education even in the context of publicly funded vaccination programs.

Finally, we found that HPV vaccine refusal was highest among girls whose parents/guardians had also refused the MMR, DTP, hepatitis B, and meningococcal conjugate vaccines. These findings are consistent with previous studies on factors influencing HPV vaccine acceptance $[16,23,41]$ suggesting that caregiver knowledge, values and beliefs regarding vaccination may be a universal determinant of HPV vaccine acceptance, independent of girls' medical history, health care utilization, and characteristics of the environment in which she lives.

Our study has a number of strengths, including that it benefits from a large, representative sample and validated HPV vaccination data. Nevertheless, there are also a number of limitations that should be considered. First, the factors available for analysis were restricted to those captured by administrative health databases and the Canadian
Census. As a result, residual confounding may have been introduced if important determinants were not included in the analysis. In addition, given that we did not have information on individual-level deprivation, we cannot be certain that area deprivation is a determinant of HPV vaccine refusal independent of individual-level deprivation. However, our analysis did account for one important component of individual-level deprivation (i.e., family income), and area deprivation remained an independent predictor. Another limitation is that a girl's vaccination status would have been misclassified as "refused" if she moved outside of the 21 participating health units during study follow-up and subsequently received her first dose of the vaccine elsewhere. However, given most girls who initiate their vaccination series do so within the first few months following cohort entry, this proportion is likely to be very low. It is also important to recognize that, given the high prevalence of the outcome (vaccine refusal), the odds ratios overestimate the risk ratios. Finally, the generalizability of our results to jurisdictions is unknown.

\section{Conclusion}

Our study highlights the importance of including characteristics of both the girl and her environment when carrying out studies on HPV vaccination. In addition, our results indicate that certain medical conditions should be further investigated as potential determinants of HPV vaccine use and as potential confounders of the 
effects of HPV vaccination. Finally, our results support the positive impact physicians may have on HPV vaccine use, even in the context of a publicly funded system, suggesting physicians could play a particularly valuable role in HPV vaccine educational efforts.

\section{Additional files}

\section{Additional file 1: Diagnostic Codes used to identify medical conditions. \\ Additional file 2: 2006 Canada Census variables considered as potential health unit-level determinants of HPV vaccine refusal. \\ Additional file 3: Health unit characteristics comprising the Pampalon index.}

\section{Competing interests}

The authors declare that they have no competing interests. The sources of funding for this study had no role in the design, execution, or reporting of this study, nor in the decision to submit this manuscript for publication.

\section{Authors' contributions}

OR was involved in the conception and design of this study, carried out the statistical analyses, interpreted the results, and prepared the first draft of the manuscript. LMS participated in acquiring the data and funding for this study, contributed to the analysis and interpretation of the results, and critically reviewed the manuscript. BAL played a major role in the design, execution, and interpretation of the study, and critically reviewed the manuscript. LC supported OR in the analysis and interpretation of the data and critically reviewed the manuscript. LEL was responsible for acquiring data and funding for the study, played a major role in the design, execution, and interpretation of the study, and critically reviewed the manuscript. All authors have given final approval of the manuscript and agree to be accountable for all aspects of the work.

\section{Acknowledgements}

This study was funded by a grant from the Ontario Ministry of Health and Long-Term Care Drug Innovation Fund (MOHLTC-DIF). The sponsor had no role in the design, conduct, analysis, interpretation, preparation, review or approval of the manuscript, or the decision to submit the manuscript for publication. This study was also supported by the Institute for Clinical Evaluative Sciences (ICES), which is funded by an annual grant from the Ontario Ministry of Health and Long-Term Care (MOHLTC). The opinions, results and conclusions reported in this paper are those of the authors and are independent from the funding sources. No endorsement by ICES or the Ontario MOHLTC is intended or should be inferred.

\section{Author details}

'Department of Public Health Sciences, Queen's University, 21 Arch Street, Room 313, Kingston, Ontario, Canada. ²Department of Epidemiology, Biostatistics, and Occupational Health, McGill University, Montreal, Quebec, Canada.

Received: 18 March 2014 Accepted: 1 October 2014

Published: 8 October 2014

\section{References}

1. Syrjanen K, Hakama M, Saarikoski S, Vayrynen M, Yliskoski M, Syrjanen S, Kataja V, Castren O: Prevalence, incidence, and estimated life-time risk of cervical human papillomavirus infections in a nonselected Finnish female population. Sex Transm Dis 1990, 17(1):15-19.

2. Patel $H$, Wagner $M$, Singhal $P$, Kothari S: Systematic review of the incidence and prevalence of genital warts. BMC Infect Dis 2013, 13:39.

3. Gall SA: Female genital warts: global trends and treatments. Infect Dis Obstet Gynecol 2001, 9:149-154.

4. Wiley DJ, Douglas J, Beutner K, Cox T, Fife K, Moscicki AB, Fukumoto L: External genital warts: diagnosis, treatment, and prevention. Clin Infect Dis 2002, 35:S210-S224.
5. Munoz N, Bosch FX, Castellsaque X, Diaz M, de Sanjose S, Hammouda D, Shah $\mathrm{KV}$, Meijer CJ: Against which human papillomavirus types shall we vaccinate and screen? The International Perspective. Int J Cancer 2004, 111(2):278-285.

6. Smith JS, Lindsay L, Hoots B, Keys J, Franceschi S, Winer R, Clifford GM: Human papillomavirus type distribution in invasive cervical cancer and high-grade cervical lesions: a meta-analysis update. Int J Cancer 2007, 121(3):621-632.

7. Brisson M, Van De Velde N, Boily MC, De Wals P: The health and economic burden of HPV infection, genital warts, cervical dysplasia and cervical cancer in Canada. Can J Infect Dis Med Microbiol 2006, 17(6):348-349.

8. Parry J: Vaccinating against cervical cancer. Bull World Health Organ 2007, 85(2):89-90

9. Colucci R, Hryniuk W, Savage C: HPV vaccination programs in Canada: Are we hitting the mark? Report Card on Cancer in Canada. Available at http://www.canceradvocacy.ca/reportcard/2008/HPV\%20Vaccination\% 20Programs\%20in\%20Canada.pdf (2008). Accessed September 2013.

10. Shearer BD: HPV Vaccination: Understanding the Impact on HPV disease. Winnipeg, MB: National Collaborating Centre for Infectious Diseases; 2011:1-18.

11. Medical Officer of Health. Toronto Public Health: Human Papillomavirus Vaccination Program Update (2008). Available at http:/www.toronto.ca/legdocs/ mmis/2008/hl/decisions/2008-09-18-hl17-dd.pdf (2011). Accessed September 2013.

12. Markowitz LE, Tsu V, Deeks SL, Cubie H, Wang SA, Vicari AS, Brotherton JML: Human Papillomavirus Vaccine Introduction - The First Five Years. Vaccine 2012, 30(Suppl 5):F139-F148.

13. Larson HJ, Jarrett C, Eckersberger E, Smith DMD, Paterson P: Understanding vaccine hesitancy around vaccines and vaccination from a global perspective: A systematic review of published literature, 2007-2012. Vaccine 2014, 32:2150-2159.

14. Kumar S, Quinn SC, Kim SH, Musa D, Hilyard KM, Freimuth VS: The social ecological model as a framework for determinants of 2009 H1N1 influenza vaccine uptake in the United States. Health Educ Behav 2012, 39(2):229-243.

15. Reiter PL, Brewer NT, Gottlieb SL, McRee A-L, Smith JS: Parents' health beliefs and HPV vaccination of their adolescent daughters. Soc Sci Med 2009, 69(3):475-480.

16. Brewer NT, Fazekas KI: Predictors of HPV vaccine acceptability: a theoryinformed, systematic review. Prev Med 2007, 45(2-3):107-114.

17. Ontario Ministry of Health and Long-Term Care: Ontario's HPV Vaccination Program. Available at http://www.health.gov.on.ca/en/ms/hpv/immunize. aspx (2013) Accessed May 2013.

18. Juurlink D, Preyra C, Croxford R, Chong A, Austin P, Tu J, Laupacis A: Canadian Institute for Health Information Discharge Abstract Database: a validation study. Institute for Clinical Evaluative Sciences; 2006.

19. Improving Health Care Data in Ontario. ICES Investigative Report. Toronto: Institute for Clinical Evaluative Sciences; 2005.

20. Institute for Clinical Evaluative Sciences: Data \& Programming at ICES. Outside ICES. Available from https://datadictionary.ices.on.ca/Applications/ DataDictionary/Default.aspx.

21. Iron K: Moving Toward a Better Health Data System for Ontario. Toronto: Institute for Clinical Evaluative Sciences; 2006.

22. Iron K, Manuel DG: Quality Assessment of Administrative Data: An Opportunity for Enhancing Ontario's Health Data. ICES Investigative Report. Toronto: Institute for Clinical Evaluative Sciences; 2007.

23. Smith LM, Brassard P, Kwong JC, Deeks SL, Ellis AK, Levesque LE: Factors associated with initiation and completion of the quadrivalent human papillomavirus vaccine series in an Ontario cohort of Grade 8 girls. BMC Public Health 2011, 11:645.

24. Lim WT, Sears K, Smith LM, Liu G, Levesque LE: Evidence of effective delivery of the human papillomavirus (HPV) vaccine through a publicly funded, school-based program: the Ontario Grade 8 HPV vaccine cohort study. BMC Public Health 2014, In Press.

25. Ontario Ministry of Health and Long-Term Care: Immunization Management Protocol. Available at http://www.health.gov.on.ca/en/pro/programs/publichealth/ oph_standards/docs/immunization_management.pdf (2013) Accessed July 2013.

26. Smith LM, Levesque LE, Nasr M, Perry AG: Validity of the Immunization Record Information System (IRIS) database for epidemiologic studies of the human papillomavirus (HPV) vaccine. Can J Clin Pharmacol 2010, 17(1):e90-e127. Abstract.

27. Statistics Canada: Census of Canada. Available at http://www12.statcan.gc. ca/census-recensement/index-eng.cfm (2013) Accessed January 2013.

28. Lévesque LE, Smith LM, Perry AG, Nsar M, Hogan ML, Martin A, Monreal M: The Ontario Grade 8 HPV Vaccine Cohort Study: A Feasibility and Validity Evaluation. Toronto, ON: Ontario Vaccine Sciences Symposium; 2011. 
29. Pampalon R, Hamel D, Gamache P, Raymond G: A deprivation index for health planning in Canada. Chronic Dis Can 2009, 29(4):178-191.

30. Hatcher L: Step by Step Approach to Using the SAS System for Factor Analysis and Structural Equation Modelling. Cary, NC: SAS Institute Inc.; 1994.

31. Eheman C, Henley SJ, Ballard-Barbash R, Jacobs EJ, Schymura MJ, Noone AM, Pan L, Anderson RN, Fulton JE, Kohler BA, Jemal A, Ward E, Plescia M, Ries $L A$, Edwards BK: Annual report to the nation on the status of cancer, 1975-2008, featuring cancers associated with excess weight and lack of sufficient physical activity. Cancer 2012, 18(9):2338-2366.

32. Melyn MA, White DT: Mental and developmental milestones of noninstitutionalized Down's Syndome children. Pediatrics 1973, 52:542-545.

33. Hurlbutt KS: Experiences of parents who homeschool their children with autism spectrum disorders. Focus Autism Other Dev Disabl 2011, 26(4):239-249.

34. Pownall JD, Jahoda A, Hastings RP: Sexuality and sex education of adolescents with intellectual disability: mother's attitudes, experiences, and support needs. I Intellect Dev Disabil 2012, 50(2):140-154.

35. Cuskelly M, Bryde R: Attitudes towards the sexuality of adults with an intellectual disability: parents, support staff, and a community sample. $J$ Intellect Dev Disabil 2004, 29:255-264.

36. Smith N, Harrell S: Sexual abuse of children with disabilities: a national snapshot. Issue Brief, Center on Victimization and Safety, U.S. VERA Institute of Justice. (2013). Available at: http://www.vera.org/sites/default/files/ resources/downloads/sexual-abuse-of-children-with-disabilities-nationalsnapshot.pdf Accessed December 2012.

37. Zimet GD: Improving adolescent health: Focus on HPV vaccine acceptance. J Adolesc Health 2005, 37:S17-\$23.

38. Gonik B: Strategies for fostering HPV vaccine acceptance. Infect Dis Obstet Gynecol 2006, 36797:1-4

39. Mackillop WJ, Zhang-Salomons J, Boyd CJ, Groome PA: Associations between community income and cancer incidence in Canada and the United States. Cancer 2000, 89(901):912.

40. Ng E, Wilkins R, Fung MF, Berthelot JM: Cervical cancer mortality by neighbourhood income in urban Canada from 1971 to 1996. CMAJ 2010, 170(10):1545-1549.

41. Ogilvie G, Anderson M, Marra F, McNeil S, Pielak K, Dawar M, Mclvor M, Ehlen T, Dobson S, Money D, Patrick DM, Naus M: A population-based evaluation of a publicly funded, school-based HPV vaccine program in British Columbia, Canada: parental factors associated with HPV vaccine receipt. PloS Med 2010, 7(5):e1000270.

doi:10.1186/1471-2458-14-1047

Cite this article as: Remes et al:: Individual- and Regional-level determinants of Human Papillomavirus (HPV) vaccine refusal: the Ontario Grade 8 HPV vaccine cohort study. BMC Public Health 2014 14:1047.

\section{Submit your next manuscript to BioMed Central and take full advantage of:}

- Convenient online submission

- Thorough peer review

- No space constraints or color figure charges

- Immediate publication on acceptance

- Inclusion in PubMed, CAS, Scopus and Google Scholar

- Research which is freely available for redistribution

Submit your manuscript at www.biomedcentral.com/submit
Biomed Central 